\title{
Case series of four secondary mucormycosis infections in COVID-19 patients, the Netherlands, December 2020 to May 2021
}

Jochem B Buil1, Arthur R H van Zanten², Robbert G Bentvelsen ${ }^{3,4}$, Tom A Rijpstra 5 , Bram Goorhuis 6 , Sanne van der Voort ${ }^{6}$, Linda J. Wammes $^{4}$, Jeroen A Janson 7 , Max Melchers ${ }^{2}$, Moniek Heusinkveld ${ }^{8}$, Willem J G Melchers ${ }^{1}$, Ed J Kuijper ${ }^{4,9}$, Paul E Verweij, ${ }^{1,9}$

1. Department of Medical Microbiology, Radboud University Medical Center, and Radboudumc-CWZ Center of Expertise for Mycology, Nijmegen, the Netherlands

2. Department of Intensive Care, Gelderse Vallei Hospital, Ede, the Netherlands

3. Microvida Laboratory for Microbiology, Amphia Hospital, Breda, the Netherlands

4. Department of Medical Microbiology, Leiden University Medical Center, Leiden, the Netherlands

5. Department of Intensive Care, Amphia Hospital, Breda, the Netherlands

6. Department of Infectious Diseases, Amsterdam University Medical Center, Amsterdam, the Netherlands

7. Department of Intensive Care, Leiden University Medical Center, Leiden, the Netherlands

8. Department of Medical Microbiology, Gelderse Vallei Hospital, Ede, the Netherlands

9. Center for Infectious Disease Research, Diagnostics and Laboratory Surveillance National Institute for Public Health and the Environment (RIVM), Bilthoven, the Netherlands

Correspondence: Paul E Verweij (paul.verweij@radboudumc.nl)

Citation style for this article:

Buil Jochem B, van Zanten Arthur R H, Bentvelsen Robbert G, Rijpstra Tom A, Goorhuis Bram, van der Voort Sanne, Wammes Linda J., Janson Jeroen A, Melchers Max, Heusinkveld Moniek, Melchers Willem J G, Kuijper Ed J, Verweij Paul E. Case series of four secondary mucormycosis infections in COVID-19 patients, the Netherlands, December 2020 to May 2021. Euro Surveill. 2021;26(23):pii=2100510. https://doi.org/10.2807/1560-7917.ES.2021.26.23.2100510

We describe four secondary fungal infections caused by Mucorales species in COVID-19 patients. Three COVID-19 associated mucormycosis (CAM) occurred in ICU, one outside ICU. All were men aged > 50 years, three died. Clinical presentations included pulmonary, rhino-orbital cerebral and disseminated infection. Infections occurred in patients with and without diabetes mellitus. CAM is an emerging disease and our observations underscore the need to be aware of invasive mucormycosis, including in COVID-19 patients without (poorly controlled) diabetes mellitus and outside ICU.

Ca $15 \%$ of patients with coronavirus disease (COVID-19) admitted to the hospital require mechanical ventilation in the intensive care unit (ICU) $[1,2]$. Secondary fungal infections were described in critically-ill ventilated COVID-19 patients in the initial reports from Wuhan, and subsequent studies from Europe reported variable frequencies of $3 \%$ to $33 \%$ of COVID-19 associated pulmonary aspergillosis (CAPA), with reports of azole resistant strains [3-7]. CAPA was associated with a $50 \%$ mortality rate and is mainly caused by Aspergillus fumigatus [8]. Recently, secondary fungal infections caused by Mucorales in critically ill COVID-19 patients were reported from India, with mortality rates ranging between $24.3 \%$ and $81 \%$ depending on the clinical manifestation $[9,10]$.

We describe a series of four cases of secondary infection with Mucorales in real-time PCR confirmed
COVID-19 patients in the Netherlands diagnosed between December 2020 and May 2021, of which three developed the infection during ICU admission and three died.

\section{Case series}

Case 1 is a man in his mid-6os who was admitted to the ICU of a general hospital 8 days after the first COVID-19 symptoms. He had no underlying diseases and received a single dose of tocilizumab and dexamethasone over 10 days starting on day 1 of ICU admission. The patient was clinically stable until he developed bilateral consolidations with no typical radiologic fungal infection signs on computed tomography (CT) on day 9 at ICU. The same day oropharynx and sputum cultures were positive with Rhizopus microsporus and a bronchoscopy was performed, with $R$. microsporus growing in a BAL culture. Treatment with liposomal amphotericin B was initiated on day 13 , and after clinical deterioration and worsening consolidations on chest CT posaconazole was added on day 19. The patient is currently continuing antifungal treatment, while receiving mechanical ventilation in the ICU.

Case 2 is a man in his late 50 s who was admitted to the ICU of a University Medical Centre 6 days after onset of COVID-19 symptoms. The patient had no underlying diseases and was treated with dexamethasone from day 1 of ICU admission and received one dose of tocilizumab. Voriconazole was started because of positive $A$. fumigatus sputum and BAL cultures on day 6 in 


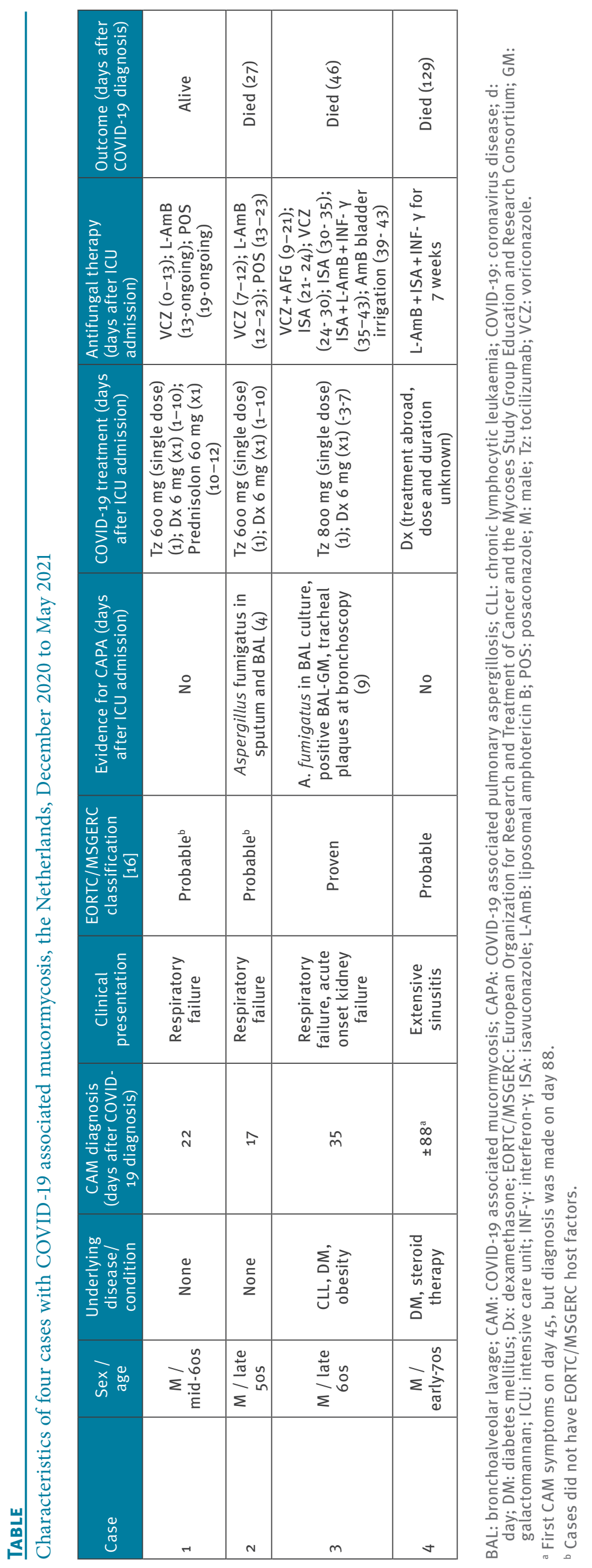


ICU, but a sputum culture obtained on day 9 was also positive with Lichtheimia ramosa. CT scan showed pulmonary cavities and a reversed halo-sign. Repeat sputum and cultures from three BALs were subsequently positive with $L$. ramosa, and voriconazole therapy was changed to liposomal amphotericin B and posaconazole. The clinical condition deteriorated, and the patient died on day 21 of ICU admission. An autopsy was not performed.

Case 3 is a man in his late 6os with known chronic lymphocytic leukaemia that had been stable for 8 years. The patient was also diagnosed with diabetes mellitus and obesity. He had COVID-19, was admitted to the ICU of a general hospital for mechanical ventilation because of progressive respiratory failure, and was treated from day 1 on ICU with dexamethasone, later changed to prednisone and a single dose of tocilizumab on day 1 . On day 10 of ICU admission, respiratory deterioration and acute-onset kidney failure occurred. Treatment with voriconazole and micafungin was initiated due to positive sputum cultures with $A$. fumigatus, but on day $21, R$. microsporus was cultured from sputum. A subsequent lung biopsy was negative, but antifungal therapy was changed to isavuconazole, and later liposomal amphotericin B was added. Subsequent CT scans on day 30, showed no evidence for sinusitis and cerebral lesions, but indicated progression of pulmonary lesions and dissemination to the kidneys, and microscopy showed non-septate hyphae in the patient's urine. The patient died and disseminated mucormycosis was confirmed at autopsy.

Case 4 is a man in his early 70 sho had been treated for COVID-19, including systemic corticosteroids, in a hospital abroad. Within a month after discharge, he was re-admitted in the same country for treatment of a cerebrovascular stroke causing left-sided paralysis. During this admission, his known diabetes mellitus was poorly controlled. Shortly after returning to the Netherlands, he was referred to a University Medical Centre with fever and swelling of his right eye, with loss of vision, caused by extensive sinusitis with intracranial necrosis and infarction. Orbital pus was positive for $R$. arrhizus. Surgical debridement and treatment with liposomal amphotericin B, isavuconazole and interferon- $\gamma$ did not successfully contain the infection, and the patient died.

The table provides an overview of the cases and the treatment received and clinical outcome.

\section{Discussion}

Mucormycosis is a rare invasive fungal disease often seen in immunocompromised individuals, in patients with diabetic ketoacidosis and in patients with concomitant use of steroids [11]. Cases of mucormycosis are now being reported in patients with severe COVID19, associated with uncontrolled diabetes mellitus and systemic corticosteroid therapy [9]. Numerous cases of COVID-19 associated mucormycosis (CAM) are reported from India, which is known to have a high burden of (poorly regulated) diabetes mellitus $[9,12]$. However, a recent cohort of 38 CAM cases was reported from outside India, including cases from Europe, the Middle East, North and South America, with an estimated prevalence of $0.3 \%$ to $0.8 \%$ [10]. Common characteristics of patients with CAM included the presence of a risk factor in $95 \%$ of patients, mainly diabetes mellitus (82.5\%). In most patients, the diabetes mellitus was poorly or uncontrolled $(80.3 \%)$, and $75 \%$ of patients received systemic corticosteroids. CAM presented mainly as rhino-orbital cerebral mucormycosis, especially in patients with diabetes mellitus, and pulmonary infection, while disseminated disease was uncommon [10]. Furthermore, due to surgical interventions in patients with rhino-orbital cerebral mucormycosis, loss of vision was common in survivors [10].

We report four CAM cases, one confirmed, three probable, all of which occurred in men aged between in their late $50 \mathrm{~s}$ and mid-70s, receiving corticosteroids, while only two had underlying diseases. CAM was characterised by a variety of clinical presentations, including pulmonary, rhino-orbital cerebral and disseminated infection. CAM occurred in COVID-19 cases admitted to the ICU and in one non-ICU case, cases without diabetes mellitus, and cases with concomitant CAPA. Furthermore, cases were observed both in academic hospitals as well as general hospitals. CT-scan was unhelpful in most cases to diagnose CAM due to extensive COVID-19 lesions, although in one case a reversed halo-sign was observed. In the three ICU cases, Mucorales were first cultured in sputum, routinely collected in Dutch ICUs from patients receiving selective decontamination of the gastrointestinal tract, who are screened for Gram-negative bacteria and yeasts. In all cases, subsequent diagnostic interventions ( $2 \mathrm{x}$ $B A L$ and lung biopsy) were performed to confirm CAM diagnosis. CAM diagnosis may be difficult due to the lack of a specific biomarker and the limited availability of molecular detection tests of Mucorales in clinical microbiology laboratories. Despite appropriate antifungal therapy, three of four cases died, while one is currently continuing antifungal treatment in the ICU.

Three of the cases described here had received the anti-interleukin (IL)-6 receptor monoclonal antibody tocilizumab, but the role of immunotherapy as risk factor for developing secondary invasive fungal infections, including mucormycosis, remains unclear [13]. Reducing tissue damage through dampening hyperimmune reactivity, such as cytokine storm syndrome, may lower the risk for invasive mycoses, while at the same time, immunomodulation interventions might increase host susceptibility to invasive fungal disease [14].

Our observations underscore the need to be aware of invasive mucormycosis developing in COVID-19 patients, especially when receiving corticosteroids, including patients without (poorly controlled) diabetes mellitus and outside the ICU. Positive cultures with 
Mucorales from the sputum of other respiratory tract samples should be considered relevant and prompt a diagnostic workup, also when cultures are recovered in combination with Aspergillus. An aggressive approach is recommended when invasive mucormycosis is diagnosed, including early surgery and targeted antifungal treatment [15].

\section{Conflict of interest}

None declared.

\section{Authors' contributions}

Arthur van Zanten, Tom Rijpstra, Bram Goorhuis, Sanne van der Voort, Jeroen Janson and Max Melchers have been in charge of the four patients, collecting the data and the informed consent.

Robbert Bentvelsen, Linda Wammes and Moniek Heusinkveld are working in the clinical microbiology laboratory and performed routine microbiology testing and consultation.

Jochem Buil, Willem Melchers and Paul Verweij performed molecular testing and in vitro susceptibility testing in the national mycology reference laboratory.

Ed Kuijper and Paul Verweij assess public health aspects of COVID-19 related mycoses in the National Institute for Public Health and the Environment (RIVM).

Jochem Buil and Paul Verweij wrote the manuscript and all authors critically revised the manuscript and approved the final version.

\section{References}

1. Docherty $A B$, Harrison EM, Green CA, Hardwick HE, Pius R, Norman L, et al. , ISARIC 4 C investigators. Features of 20133 UK patients in hospital with covid-19 using the ISARIC WHO Clinical Characterisation Protocol: prospective observational cohort study. BMJ. 2020;369:m1985. https://doi.org/10.1136/ bmj.m1985 PMID: 32444460

2. Richardson S, Hirsch IS, Narasimhan M, Crawford JM, McGinn T, Davidson KW, et al. , the Northwell COVID-19 Research Consortium. Presenting Characteristics, Comorbidities, and Outcomes Among 5700 Patients Hospitalized With COVID-19 in the New York City Area. JAMA. 2020;323(20):2052-9. https:// doi.org/10.1001/jama.2020.6775 PMID: 32320003

3. Alanio A, Dellière $S$, Fodil $S$, Bretagne $S$, Mégarbane $B$. Prevalence of putative invasive pulmonary aspergillosis in critically ill patients with COVID-19. Lancet Respir Med. 2020;8(6):e48-9. https://doi.org/10.1016/S22132600(20)30237-X PMID: 32445626

4. White PL, Dhillon R, Cordey A, Hughes H, Faggian F, Soni S, et al. A national strategy to diagnose COVID-10 associated invasive fungal disease in the ICU. Clin Infect Dis. 2020;ciaa1298. PMID: 32860682

5. Bartoletti M, Pascale R, Cricca M, Rinaldi M, Maccaro A, Bussini L, et al. Epidemiology of invasive pulmonary aspergillosis among COVID-19 intubated patients: a prospective study. Clin Infect Dis. 2020; ciaa1065. PMID: 32719848

6. Sarrazyn C, Dhaese S, Demey B, Vandecasteele S, Reynders $M$, Van Praet JT. Incidence, risk factors, timing, and outcome of influenza versus COVID-19-associated putative invasive aspergillosis. Infect Control Hosp Epidemiol. 2020;1-2. PMID: 32900411

7. van Arkel ALE, Rijpstra TA, Belderbos HNA, van Wijngaarden P, Verweij PE, Bentvelsen RG. COVID-19-associated pulmonary aspergillosis. Am J Respir Crit Care Med. 2020;202(1):132-5. https://doi.org/10.1164/rccm.202004-1038LE PMID: 32396381

8. Chong WH, Neu KP. The Incidence, Diagnosis, and Outcomes of COVID-19-associated Pulmonary Aspergillosis (CAPA): A
Systematic Review. J Hosp Infect. 2021:S0195-6701(21)00163-8. https://doi.org/10.1016/j.jhin.2021.04.012

9. John TM, Jacob CN, Kontoyiannis DP. When Uncontrolled Diabetes Mellitus and Severe COVID-19 Converge: The Perfect Storm for Mucormycosis. J Fungi (Basel). 2021;7(4):298. https://doi.org/10.3390/jof7040298 PMID: 33920755

10. Hoenigl M, Seidel D, Carvalho A, Rudramurthy SM, Arastehfar A, Gangneux IP, et al. The Emergence of COVID-19 Associated Mucormycosis: Analysis of Cases From 18 Countries. SSRN Electronic Journal (Preprint) DOI:102139/ssrn3844587. 2021.

11. Skiada A, Pavleas I, Drogari-Apiranthitou M. Epidemiology and Diagnosis of Mucormycosis: An Update. J Fungi (Basel). 2020;6(4):E265. https://doi.org/10.3390/jof6040265 PMID: 33147877

12. Prakash H, Chakrabarti A. Epidemiology of Mucormycosis in India. Microorganisms. 2021;9(3):523. https://doi. org/10.3390/microorganisms9030523 PMID: 33806386

13. Falcone M, Tiseo G, Giordano C, Leonildi A, Menichini M, Vecchione A, et al. , Pisa COVID-19 Study Group. Predictors of hospital-acquired bacterial and fungal superinfections in COVID-19: a prospective observational study. J Antimicrob Chemother. 2021;76(4):1078-84. https://doi.org/10.1093/jac/ dkaa530 PMID: 33374002

14. van de Veerdonk FL, Brüggemann RJM, Vos S, De Hertogh G, Wauters J, Reijers MHE, et al. COVID-19-associated Aspergillus tracheobronchitis: the interplay between viral tropism, host defence, and fungal invasion. Lancet Respir Med. 2021;S22132600(21)00138-7. PMID: 34051176

15. Cornely OA, Alastruey-Izquierdo A, Arenz D, Chen SCA Dannaoui E, Hochhegger B, et al. , Mucormycosis ECMM MSG Global Guideline Writing Group. Global guideline for the diagnosis and management of mucormycosis: an initiative of the European Confederation of Medical Mycology in cooperation with the Mycoses Study Group Education and Research Consortium. Lancet Infect Dis. 2019;19(12):e40521. https://doi.org/10.1016/S1473-3099(19)30312-3 PMID: 31699664

16. Donnelly JP, Chen SC, Kauffman CA, Steinbach WJ, Baddley JW, Verweij PE, et al. Revision and Update of the Consensus Definitions of Invasive Fungal Disease From the European Organization for Research and Treatment of Cancer and the Mycoses Study Group Education and Research Consortium. Clin Infect Dis. 2020;71(6):1367-76. https://doi.org/10.1093/cid/ ciz1008 PMID: 31802125

\section{License, supplementary material and copyright}

This is an open-access article distributed under the terms of the Creative Commons Attribution (CC BY 4.0) Licence. You may share and adapt the material, but must give appropriate credit to the source, provide a link to the licence and indicate if changes were made.

Any supplementary material referenced in the article can be found in the online version.

This article is copyright of the authors or their affiliated institutions, 2021. 